\title{
SOME REMARKS ON THE UNIQUE FACTORIZATION IN CERTAIN SEMIGROUPS OF CLASSICAL $L$-FUNCTIONS
}

\author{
Jerzy Kaczorowski, Giuseppe Molteni \& Alberto Perelli
}

To Jean-Marc Deshouillers, with friendship and respect

\begin{abstract}
In this note we investigate problems related to the unique factorization of some semigroups of classical $L$-functions. The semigroups of Artin and automorphic $L$-functions as well as the semigroup generated by the Hecke $L$-functions of finite order are studied. The main result of the paper shows that in the latter semigroup the unique factorization into primitive elements does not hold. This closes a possible way of attacking the famous Dedekind conjecture concerning the divisibility of the Dedekind zeta functions.

Keywords: unique factorization of $L$-functions, Artin $L$-functions, automorphic $L$-functions,
\end{abstract} Hecke $L$-functions, Selberg class, Dedekind conjecture.

\section{Introduction}

A well known result by Ram Murty [9] states that the Selberg orthonormality conjecture (SOC for short) for the Selberg class $\mathcal{S}$ implies the Artin conjecture on the holomorphy of the Artin $L$-functions (see below for definitions and other information). A perhaps less known but interesting statement in the same paper is that the unique factorization conjecture for $\mathcal{S}$ (UF for short) implies the Dedekind conjecture asserting that the Riemann zeta function $\zeta(s)$ always divides the Dedekind zeta function $\zeta_{K}(s)$ of an algebraic number field $K$ (note that SOC implies UF and that the Artin conjecture implies the Dedekind conjecture, see e.g. Chapter 7 of Murty-Murty [10]). Ram Murty's argument is as follows. Let $\bar{K}$ be the normal closure over $\mathbb{Q}$ of $K$. By the Aramata-Brauer theorem (see (2-1) below), both $\zeta(s)$ and $\zeta_{K}(s)$ divide $\zeta_{\bar{K}}(s)$, in the sense that there exist entire functions $F, G \in \mathcal{S}$ such that $\zeta_{\bar{K}}(s)=\zeta(s) F(s)$ and $\zeta_{\bar{K}}(s)=\zeta_{K}(s) G(s)$. Therefore $\zeta(s) F(s)=\zeta_{K}(s) G(s)$, and hence $\zeta(s) \mid \zeta_{K}(s)$ by UF, since $\zeta(s)$ is primitive and has a pole at $s=1$. However, such an argument apparently requires the non-vanishing at $s=1$ of the functions in $\mathcal{S}$, otherwise one cannot a priori exclude situations like: $\zeta_{K}(s)$ is primitive and $G(s)=\zeta(s) H(s)$ with $H(s)$ primitive and $H(1)=0$ (and hence $F(s)=\zeta_{K}(s) H(s)$ ). Since at present the 
non-vanishing at $s=1$ is not known in the general framework of $\mathcal{S}$, Ram Murty's statement apparently requires an additional non-vanishing hypothesis (unfortunately, the uncorrect proof has been reported also on p.183 of Murty-Murty [10] and in Proposition 4.2 of $[6]$ ).

We refer to the survey papers by Kaczorowski [5], Kaczorowski-Perelli [6] and Perelli [12], [13] for the definition of the Selberg class $\mathcal{S}$ and for its basic properties. Here we recall only that $\mathcal{S}$ is, roughly speaking, the multiplicative semigroup of the Dirichlet series

$$
F(s)=\sum_{n=1}^{\infty} \frac{a_{F}(n)}{n^{s}}
$$

with analytic continuation, a functional equation of Riemann's type and an Euler product, that every function $F \in \mathcal{S}$ has a factorization into primitive functions (the irreducible elements of the semigroup $\mathcal{S}$ ) and that most of the classical $L$-functions belong to $\mathcal{S}$ (at least modulo classical conjectures). Moreover, as anticipated above, the following important conjectures are expected to hold:

(unique factorization, UF) the factorization of every $F \in \mathcal{S}$ into primitive functions is unique;

(Selberg orthonormality conjecture, SOC) for primitive $F, G \in \mathcal{S}$ we have

$$
\sum_{p \leqslant x} \frac{a_{F}(p) \overline{a_{G}(p)}}{p}=\delta_{F, G} \log \log x+o(\log \log x)
$$

where $\delta_{F, G}=1$ if $F(s)=G(s)$ and $\delta_{F, G}=0$ otherwise. As remarked above, it is well known that SOC implies UF.

We use the following notation. We denote by $\langle\mathbf{U}\rangle$ the multiplicative semigroup generated by a set $\mathbf{U}$, and by $(\mathbf{U})$ the group of quotients of $\langle\mathbf{U}\rangle . L_{K}(s, \chi)$ denotes the Hecke $L$-functions associated with the algebraic number field $K ; \chi$ is a Hecke character of finite or infinite order, and $L_{K}(s, \chi)$ is of finite or infinite order accordingly. $\mathbf{H}$ and $\mathbf{H}_{f}$ denote the sets of the Hecke $L$-functions and of the Hecke $L$-functions of finite order, respectively. Art denotes the set of the Artin $L$-functions $L(s, \rho, K / k)$, while Aut denotes the set of the automorphic $L$-functions $L(s, \rho)$. Moreover, the irreducible elements of a semigroup of $L$-functions are called primitive functions. The following characterization is very easy to prove:

a semigroup of $L$-functions has unique factorization if and only if the logarithms of the primitive functions are linearly independent over $\mathbb{Q}$.

A closer look at the Aramata-Brauer theorem, see (2.1) below, shows that the above reported argument by Ram Murty can be adapted to suitable semigroups of $L$-functions, smaller than the Selberg class, where the non-vanishing hypothesis can be proved. For example one can prove that

if the semigroup $\left\langle\mathbf{H}_{f}\right\rangle$ has unique factorization, then the Dedekind conjecture is true. 
Such a result is tempting, since $\left\langle\mathbf{H}_{f}\right\rangle$ is not an axiomatically defined semigroup like $\mathcal{S}$, and hence one should to be able to decide if the unique factorization holds for $\left\langle\mathbf{H}_{f}\right\rangle$. Unfortunately, it turns out that the unique factorization does not hold for $\left\langle\mathbf{H}_{f}\right\rangle$. Essentially, this is due to the existence of primitive functions in $\left\langle\mathbf{H}_{f}\right\rangle$ which can be factorized in larger semigroups. This phenomenon also explains why there is no incompatibility between the classical Artin conjecture and the distinct zeros conjecture from the Selberg class theory. Indeed, roughly speaking, on the one hand the distinct zeros conjecture asserts that distinct primitive functions in $\mathcal{S}$ have no common zeros. On the other hand, the Artin conjecture states that the right hand side of the Artin-Brauer theorem (2.2) below is entire, even if some of the integers $m_{j}$ are negative. These two conjectures would be incompatible if the primitive functions of $\left\langle\mathbf{H}_{f}\right\rangle$ remain primitive in $\mathcal{S}$, but the above phenomenon shows that they may well be coherent.

In this note we investigate some problems related to the unique factorization of some semigroups of classical $L$-functions. Perhaps the results we get are already known to experts in algebraic number theory; however, we couldn't find references in the literature, and gathering such results in one place might anyway be useful.

Acknowledgment. This research was partially supported by the Istituto Nazionale di Alta Matematica, by a MIUR grant Cofin2004, by the Foundation for Polish Science and by KBN grant 1 PO3A 00826.

\section{Results}

We first recall very briefly some analytic properties of the Hecke, Artin and automorphic $L$-functions. The analytic properties of the Hecke $L$-functions (see e.g. Chapter 1 of [10]) are rather well understood. Indeed, the Hecke $L$-functions are known to belong to the Selberg class. Moreover, the standard zero-free region is available in this case. Special cases of Hecke $L$-functions are the Dedekind zeta functions $\zeta_{K}(s)$, and the following well known theorem by Aramata-Brauer holds: if $L / K$ is a Galois extension then

$$
\frac{\zeta_{L}(s)}{\zeta_{K}(s)}=\prod_{j} L_{K_{j}}\left(s, \chi_{j}\right)^{n_{j}}
$$

where $n_{j} \in \mathbb{N}$ and $L_{K_{j}}\left(s, \chi_{j}\right)$ are suitable Hecke $L$-functions with non-trivial characters $\chi_{j}$ of finite order. Therefore, the Dedekind conjecture follows easily in the Galois case. From the Artin reciprocity law (see Chapter 2 of [10]) we know that the Hecke $L$-functions of finite order are special cases of Artin $L$-functions. Moreover, it is also known that

$$
\mathbf{H}_{f} \subsetneq\left\langle\mathbf{H}_{f}\right\rangle \subsetneq \text { Art. }
$$

For example, every Dirichlet $L$-function $L(s, \chi)$ is a Hecke $L$-function of finite type, but in general $L(s, \chi)^{2}$ is not a Hecke $L$-function. 
The knowledge of the analytic properties of the Artin $L$-functions (see e.g. Chapter 2 of [10]) is definitely less complete than in the Hecke $L$-functions case. In fact, such $L$-functions belong to the Selberg class modulo the Artin conjecture, asserting that $L(s, \rho, K / k)$ is entire if $\rho$ does not contain the trivial representation (in which case the only singularity is a pole at $s=1$ ). Good part of our present knowledge of the analytic properties of the Artin $L$-functions comes from the following classical theorem of Artin-Brauer: if $K / k$ is a Galois extension then

$$
L(s, \rho, K / k)=\prod_{j} L_{K_{j}}\left(s, \chi_{j}\right)^{m_{j}}
$$

where $m_{j} \in \mathbb{Z}$ and $L_{K_{j}}\left(s, \chi_{j}\right)$ are suitable Hecke $L$-functions of finite order, which we may clearly assume to be primitive in $\left\langle\mathbf{H}_{f}\right\rangle$ and distinct. Moreover, $K_{j}$ are intermediate fields, with $K / K_{j}$ Galois. As already remarked above, we recall that the Artin conjecture implies the Dedekind conjecture in the general case (see Section 2 of Chapter 2 of [10]).

We refer to Iwaniec-Sarnak [4] for a survey of the analytic properties of the automorphic $L$-functions. Here we only recall that such functions belong to the Selberg class modulo the Ramanujan conjecture. Moreover, a well established theory for the Rankin-Selberg convolution is available in this case. The Langlands conjectures predict that Art $\subset$ Aut. In fact, in a sense the automorphic $L$-functions are expected to be the most general $L$-functions, according to a conjecture asserting that the Selberg class $\mathcal{S}$ coincides with Aut.

Next we sketch a heuristic argument suggesting that the unique factorization does not hold for $\left\langle\mathbf{H}_{f}\right\rangle$; precisely, assuming SOC we show by a theoretical argument that a primitive function in $\left\langle\mathbf{H}_{f}\right\rangle$ is not necessarily primitive in $\mathcal{S}$; for an unconditional explicit example we refer to (2.16)-(2.19) below. In a way, this situation is similar to Hilbert's famous example of the semigroup of the integers $n \equiv 1(\bmod 4):$ unique factorization does not hold there, and the reason is that there are non-prime integers which are irreducible elements of such a semigroup. Let $\rho \neq 1$ be irreducible and such that the Artin $L$-function $L(s, \rho, K / \mathbb{Q})$ is not a Hecke $L$-function of finite order. Then apply the Artin-Brauer theorem (2.2), with $L_{K_{j}}\left(s, \chi_{j}\right)$ primitive in $\left\langle\mathbf{H}_{f}\right\rangle$, and hence

$$
\prod_{m_{j}<0} L_{K_{j}}\left(s, \chi_{j}\right)^{-m_{j}} L(s, \rho, K / \mathbb{Q})=\prod_{m_{j}>0} L_{K_{j}}\left(s, \chi_{j}\right)^{m_{j}} .
$$

Assuming SOC, by Theorem 3.1 of Ram Murty [9] we have that $L(s, \rho, K / \mathbb{Q})$ is a primitive function of $\mathcal{S}$. Recalling that $\mathbf{H}_{f} \subset \mathcal{S}$ and regarding (2.3) as a factorization in $\mathcal{S}$, we therefore have that $L(s, \rho, K / \mathbb{Q})$ divides one of the $L_{K_{j}}\left(s, \chi_{j}\right)$ in $\mathcal{S}$, say $L_{K_{1}}\left(s, \chi_{1}\right)$. But $L(s, \rho, K / \mathbb{Q})$ is not a Hecke $L$-function, hence $L(s, \rho, K / \mathbb{Q})$ divides properly $L_{K_{1}}\left(s, \chi_{1}\right)$, which is therefore primitive in $\left\langle\mathbf{H}_{f}\right\rangle$ but not in $\mathcal{S}$. In Theorem 2 below we also give an explicit example of a function in $\left\langle\mathbf{H}_{f}\right\rangle$ with two distinct factorizations.

Now we turn to some simple facts about the semigroups $\left\langle\mathbf{H}_{f}\right\rangle,\langle\mathbf{A r t}\rangle$ and $\langle$ Aut $\rangle$. 
Fact 1. Let $K / k$ be a Galois extension and let $G=\operatorname{Gal}(K / k)$. Let $L$ be a normal extension of $\mathbb{Q}$ containing $K$. Then $L / k$ and $L / \mathbb{Q}$ are Galois extensions, and let $H=\operatorname{Gal}(L / k), F=\operatorname{Gal}(L / \mathbb{Q})$. Let $L(s, \rho, K / k)$ be an Artin $L$-functon. By the properties of the Artin $L$-functions (see Chapter 2 of [10]) we have

$$
L(s, \rho, K / k)=L\left(s, \operatorname{Lift}_{G}^{H} \rho, L / k\right)=L\left(s, \operatorname{Ind}_{H}^{F} \operatorname{Lift}_{G}^{H} \rho, L / \mathbb{Q}\right),
$$

where $\operatorname{Lift}_{G}^{H} \rho$ and Ind ${ }_{H}^{F} \rho$ denote the lift and the induction of the representation $\rho$, respectively. Hence we may restrict our considerations only to Artin $L$-functions over $\mathbb{Q}$, thus simply writing $L(s, \rho)$ for the Artin $L$-functions (when there is no confusion with the automorphic $L$-functions), the base field being always $\mathbb{Q}$. Therefore, every Artin $L$-function can be defined by an Euler product over $\mathbb{Q}$. Moreover, the product of two Artin $L$-functions is still an Artin $L$-function, and hence Art is already a semigroup:

$$
\mathbf{A r t}=\langle\mathbf{A r t}\rangle
$$

and $\left\langle\mathbf{H}_{f}\right\rangle$ is a subsemigroup of Art.

As a consequence of Fact 1, in what follows the Artin L-functions $L(s, \rho)$ will always be assumed to have base field $\mathbb{Q}$.

Fact 2. From the properties of the Artin $L$-functions (see Chapter 2 of [10]) it is well known that the primitive functions of Art are the Artin $L$-functions associated with irreducible representations. Moreover, every representation can be written in a unique way (up to equivalence) as a sum of irreducible representations. We therefore have

Art has unique factorization,

and its primitive functions are the L-functions associated with irreducible representations. Note that (2.4) could be used to prove the Dedekind conjecture by the argument reported in the Introduction. However, one would need to assume the Artin conjecture, which we already know to imply the Dedekind conjecture.

Fact 3. Take two Artin $L$-functions

$$
L\left(s, \rho_{k}\right)=\prod_{p} \prod_{j=1}^{d_{k}}\left(1-\frac{\lambda_{j, k}(p)}{p^{s}}\right)^{-1}
$$

with $\rho_{k}$ distinct and irreducible for $k=1,2$. Consider their Rankin-Selberg convolution

$$
L\left(s, \rho_{1} \otimes \bar{\rho}_{2}\right)=\prod_{p} \prod_{\substack{j=1, \ldots, d_{1} \\ i=1, \ldots, d_{2}}}\left(1-\frac{\lambda_{j, 1}(p) \overline{\lambda_{i, 2}(p)}}{p^{s}}\right)^{-1}
$$

which is still an Artin $L$-function thanks to the Artin formalism, and let $G$ be its Galois group. Suppose that $L\left(s, \rho_{1} \otimes \bar{\rho}_{2}\right)$ has a pole at $s=1$, thus $\rho_{1} \otimes \bar{\rho}_{2}$ 
contains the trivial representation $\rho_{0}$. In terms of characters we therefore have

$$
\begin{aligned}
0 & \neq<\rho_{1} \otimes \bar{\rho}_{2}, \rho_{0}>=\frac{1}{|G|} \sum_{\sigma \in G} \chi_{\rho_{1} \otimes \bar{\rho}_{2}}(\sigma) \chi_{0}(\sigma)=\frac{1}{|G|} \sum_{\sigma \in G} \chi_{\rho_{1}}(\sigma) \chi_{\bar{\rho}_{2}}(\sigma) \\
& =<\rho_{1}, \rho_{2}>
\end{aligned}
$$

which implies that the two representations contain a common factor, contradiction. Thus $L\left(s, \rho_{1} \otimes \bar{\rho}_{2}\right)$ is holomorphic at $s=1$ in this case. By the Artin-Brauer theorem and the well known analytic properties (and zero-free regions) of the Hecke $L$-functions of finite order, standard methods therefore assure that

$$
\sum_{p \leqslant x} \frac{a_{\rho_{1} \otimes \bar{\rho}_{2}}(p)}{p}=o(\log \log x)
$$

and hence, by the definition of $L\left(s, \rho_{1} \otimes \bar{\rho}_{2}\right)$,

$$
\sum_{p \leqslant x} \frac{a_{\rho_{1}}(p) \overline{a_{\rho_{2}}(p)}}{p}=o(\log \log x)
$$

if $\rho_{1}$ and $\rho_{2}$ are distinct and irreducible, where $a_{\rho}(n)$ denotes the Dirichlet coefficients of $L(s, \rho)$.

On the other hand, if $\rho_{1}=\rho_{2}=\rho$ is irreducible then the Chebotarev density theorem (see Chapter 2 of [10]) implies that

$$
\sum_{p \leqslant x} \frac{\left|a_{\rho}(p)\right|^{2}}{p} \sim \log \log x .
$$

Therefore, from (2.5) and (2.6) we have that

the orthogonality relation (1.1) holds for the semigroup Art.

Note that one could easily deduce (2.4) from (2.7), in the same way as SOC implies UF (see e.g. Section 4 of [6]). Note also that in terms of the Artin $L$-functions, relation $(2.7)$ can be expressed as follows:

$$
\begin{gathered}
\text { given two irreducible representations } \rho_{1} \text { and } \rho_{2}, L\left(s, \rho_{1} \otimes \bar{\rho}_{2}\right) \\
\text { has a simple pole at } s=1 \text { if and only if } \rho_{1}=\rho_{2} .
\end{gathered}
$$

Further, assuming the Artin conjecture one may ask if the primitive functions of Art are still primitive in $\mathcal{S}$; this is the case under the assumption of SOC, as proved by Murty [9].

Fact 4. We first recall that the product of automorphic $L$-functions is still an automorphic $L$-function, hence

$$
\text { Aut }=\langle\text { Aut }\rangle \text {. }
$$


The semigroup Aut has properties similar to Art. In fact, by the properties of the Rankin-Selberg convolution in Aut it is easy to see that

Aut has unique factorization.

Moreover, a standard exercise in prime number theory shows that under the Ramanujan conjecture the orthogonality relation (1.1) holds for the semigroup Aut. These facts are perhaps the best evidence in favour of SOC and UF for $\mathcal{S}$.

Fact 5. Consider now the set $\mathbf{H}_{f}^{K}$ of the Hecke $L$-functions of finite order defined over a fixed algebraic number field $K$ and the semigroup $\left\langle\mathbf{H}_{f}^{K}\right\rangle$ generated by $\mathbf{H}_{f}^{K}$. Given $L_{K}\left(s, \chi_{1}\right)$ and $L_{K}\left(s, \chi_{2}\right)$ in $\mathbf{H}_{f}^{K}$, we define the Rankin-Selberg convolution over $K$ as

$$
L_{K}\left(s, \chi_{1} \bar{\chi}_{2}\right)=\prod_{\mathfrak{p}}\left(1-\frac{\chi_{1}(\mathfrak{p}) \bar{\chi}_{2}(\mathfrak{p})}{N \mathfrak{p}^{s}}\right)^{-1},
$$

where $\mathfrak{p}$ runs over the prime ideals of the ring of integers of $K$ and $N \mathfrak{p}$ is its norm. Then $L_{K}\left(s, \chi_{1} \bar{\chi}_{2}\right)$ is holomorphic at $s=1$ if $\chi_{1} \neq \chi_{2}$ (since $\chi_{1} \bar{\chi}_{2} \neq \chi_{0}$, the trivial character, in this case), while it has a simple pole at $s=1$ if $\chi_{1}=\chi_{2}$ (since now $\chi_{1} \bar{\chi}_{2}=\chi_{0}$ ). Thus the functions of $\mathbf{H}_{f}^{K}$ satisfy a kind of orthonormality over $K$, and this implies by standard arguments that

$$
\left\langle\mathbf{H}_{f}^{K}\right\rangle \text { has unique factorization. }
$$

Now we turn to a more detailed discussion of $\left\langle\mathbf{H}_{f}\right\rangle$ and Art. From the Artin reciprocity law and the Artin-Brauer theorem we know that

$$
\left\langle\mathbf{H}_{f}\right\rangle \subsetneq \operatorname{Art} \subsetneq\left(\mathbf{H}_{f}\right) .
$$

In fact, the inclusions are easy, the first inequality follows from the existence of Artin $L$-functions with positive and negative exponents in (2.2), and for the second inequality it suffices to note that $1 / \zeta(s)$ is not an Artin $L$-function. Moreover, we know that Art has unique factorization, while we will prove in Theorem 2 below that factorization in $\left\langle\mathbf{H}_{f}\right\rangle$ is not unique. The following natural question therefore arises:

$$
\text { is Art the smallest unique factorization semigroup containing }\left\langle\mathbf{H}_{f}\right\rangle \text { ? }
$$

We suspect that the answer is yes. In general, given a reduced semigroup $\mathbf{S}$ (i.e. such that the units of $\mathbf{S}$ reduce to the identity, which is clearly the case of $\left\langle\mathbf{H}_{f}\right\rangle$ ) and a unique factorization semigroup $\mathbf{D}$, a divisor theory for $\mathbf{S}$ is an injective homomorphism $\partial: \mathbf{S} \rightarrow \mathbf{D}$ such that $a \mid b$ in $\mathbf{S}$ if and only if $\partial(a) \mid \partial(b)$ in $\mathbf{D}$, and for every $d \in \mathbf{D}$ there exist elements $a_{1}, \ldots, a_{n} \in \mathbf{S}$ such that $d=$ g.c.d. $\left(\partial\left(a_{1}\right), \ldots, \partial\left(a_{n}\right)\right)$. We refer to Halter-Hoch [2] for a survey of such a theory, whose better known example is probably the case of the semigroups of the principal ideals and of the ideals of the ring of integers of an algebraic number field. For example, 
the injection $i:\left\langle\mathbf{H}_{f}\right\rangle \rightarrow \mathbf{A r t}$ is not a divisor theory.

Consider indeed an Artin $L$-function not in $\left\langle\mathbf{H}_{f}\right\rangle$; by the Artin-Brauer theorem in the form (2.3) we have that

$$
\prod_{m_{j}<0} L_{K_{j}}\left(s, \chi_{j}\right)^{-m_{j}} \quad \text { divides } \prod_{m_{j}>0} L_{K_{j}}\left(s, \chi_{j}\right)^{m_{j}}
$$

in Art, but clearly not in $\left\langle\mathbf{H}_{f}\right\rangle$. We conjecture that

$$
\left\langle\mathbf{H}_{f}\right\rangle \text { has no divisor theory; }
$$

this is probably related with the previous question.

It is known that a divisor theory of a semigroup (if it exists) is uniquely defined up to semigroup isomorphism, see Clifford [1]. It is also known that the Riemann zeta function $\zeta(s)$ is primitive in the Selberg class (see e.g. [6]), hence it is primitive in $\left\langle\mathbf{H}_{f}\right\rangle$. Thus if a divisor theory of $\left\langle\mathbf{H}_{f}\right\rangle$ exists such that $\partial(\zeta(s))$ is primitive in $\mathbf{D}$, then every divisor theory of $\left\langle\mathbf{H}_{f}\right\rangle$ has this property. We remark that if the above conjecture is false and the divisor theory for $\left\langle\mathbf{H}_{f}\right\rangle$ exists and satisfies such a property, then it is not difficult to deduce the Dedekind conjecture. In fact we have

Theorem 1. Suppose that the divisor theory $\partial:\left\langle\mathbf{H}_{f}\right\rangle \rightarrow \mathbf{D}$ exists and $\partial(\zeta(s))$ is primitive in $\mathbf{D}$. Then the Dedekind conjecture holds true.

Proof. Arguing as in the Introduction, by the Aramata-Brauer theorem applied to $\zeta_{\bar{K}}(s) / \zeta(s)$ and to $\zeta_{\bar{K}}(s) / \zeta_{K}(s)$ we have

$$
\zeta(s) \prod_{j} L_{K_{j}}\left(s, \chi_{j}\right)=\zeta_{K}(s) \prod_{i} L_{K_{i}^{\prime}}\left(s, \chi_{i}^{\prime}\right)
$$

where the $L_{K_{j}}\left(s, \chi_{j}\right)$ and the $L_{K_{i}^{\prime}}\left(s, \chi_{i}^{\prime}\right)$ are Hecke $L$-functions of finite order. Since the Hecke $L$-functions do not vanish at $s=1$, the above identity implies that all the $L_{K_{j}}\left(s, \chi_{j}\right)$ and $L_{K_{i}^{\prime}}\left(s, \chi_{i}^{\prime}\right)$ are holomorphic at $s=1$. Applying the homomorphism $\partial$ and recalling that $\partial(\zeta(s))$ is primitive in the unique factorization semigroup $\mathbf{D}$, we have that $\partial(\zeta(s))$ divides one of the elements $\partial\left(L_{K_{i}^{\prime}}\left(s, \chi_{i}^{\prime}\right)\right)$ or $\partial\left(\zeta_{K}(s)\right)$. In the first case, by the divisor theory we deduce that $\zeta(s)$ divides a function $L_{K_{i}^{\prime}}\left(s, \chi_{i}^{\prime}\right)$ in $\left\langle\mathbf{H}_{f}\right\rangle$, a contradiction since the $L_{K_{i}^{\prime}}\left(s, \chi_{i}^{\prime}\right)$ 's are holomorphic at $s=1$ and the Hecke $L$-functions do not vanish at $s=1$. Hence the second case must occur, and hence by the divisor theory we deduce that $\zeta(s) \mid \zeta_{K}(s)$ in $\left\langle\mathbf{H}_{f}\right\rangle$, and the result follows.

Note that Theorem 1 gives in fact a slightly stronger form of the Dedekind conjecture, namely that the quotient $\zeta_{K}(s) / \zeta(s)$ is a product of Hecke $L$-functions of finite order. This agrees with the case of Galois extensions, but it is not clear to us if it has to be expected in the general case as well. Note also that the hypothesis that $\partial(\zeta(s))$ is primitive in $\mathbf{D}$ is important in the proof. Indeed, in order to prove a result of type of Theorem 1 one needs some control on the factorization of $\partial(\zeta(s))$ in $\mathbf{D}$. 
We finally turn to the main result of this paper, namely

Theorem 2. Unique factorization does not hold in $\left\langle\mathbf{H}_{f}\right\rangle$.

Proof. We prove that $\left\langle\mathbf{H}_{f}\right\rangle$ is not UF by constructing an Artin $L$ function belonging to $\left\langle\mathbf{H}_{f}\right\rangle$ and admitting two distinct factorizations in $\left\langle\mathbf{H}_{f}\right\rangle$.

We start by choosing a suitable group, which will became the Galois group of an extension $L / \mathbb{Q}$. Let $G=\mathrm{SL}_{2}(3)$, i.e. the group of $2 \times 2$ matrices with determinant 1 over the finite field with three elements. A presentation of $G$ is

$$
G=\left\langle a^{3}=b^{4}=(a b)^{3}=1, a b^{2} a^{-1}=b^{2}\right\rangle,
$$

where $a=\left(\begin{array}{ll}1 & 1 \\ 0 & 1\end{array}\right)$ and $b=\left(\begin{array}{rr}0 & 1 \\ -1 & 0\end{array}\right)$. The order of $G$ is 24 and the table of its irreducible characters is the following (see [3], p.288):

\begin{tabular}{|c|c|c|c|c|c|c|c|}
\hline$g$ & 1 & $c$ & $c^{2}$ & $c^{3}=b^{2}$ & $c^{4}$ & $c^{5}$ & $b \sim b^{3}$ \\
\hline$|\mathrm{Cl}(g)|$ & 1 & 4 & 4 & 1 & 4 & 4 & 6 \\
\hline$\chi_{1}$ & 1 & 1 & 1 & 1 & 1 & 1 & 1 \\
$\chi_{2}$ & 1 & $\omega$ & $\bar{\omega}$ & 1 & $\omega$ & $\bar{\omega}$ & 1 \\
$\chi_{3}$ & 1 & $\bar{\omega}$ & $\omega$ & 1 & $\bar{\omega}$ & $\omega$ & 1 \\
$\chi_{4}$ & 2 & 1 & -1 & -2 & -1 & 1 & 0 \\
$\chi_{5}$ & 2 & $\omega$ & $-\bar{\omega}$ & -2 & $-\omega$ & $\bar{\omega}$ & 0 \\
$\chi_{6}$ & 2 & $\bar{\omega}$ & $-\omega$ & -2 & $-\bar{\omega}$ & $\omega$ & 0 \\
$\chi_{7}$ & 3 & 0 & 0 & 3 & 0 & 0 & -1 \\
\hline
\end{tabular}

( $\omega$ is a primitive cubic root of 1 and $c=a b^{2}$ )

The characters $\chi_{1}, \chi_{2}$ and $\chi_{3}$ are evidently monomial. Also $\chi_{7}$ is monomial (it is induced by a non-trivial 1-dimensional representation of the derived subgroup of $G$ ). On the contrary, $\chi_{4}, \chi_{5}$ are $\chi_{6}$ are not monomial since $G$ does not contain any subgroup of order 12. Moreover, it is well known that $G$ is solvable (see [3]).

Let $H$ be the subgroup of $G$ generated by $a$ and $b^{2} . H$ is cyclic, a generator being $c=a b^{2}$, and its order is 6 . Let $\eta_{1}, \eta_{2}$ and $\eta_{3}$ be the irreducible characters of $H$ defined by $\eta_{1}(c)=-\omega, \eta_{2}(c)=-\bar{\omega}$ and $\eta_{3}(c)=-1$. The representation induced in $G$ by every $\eta_{j}$ has degree $[G: H]=4$. Using the Frobenius formula $\left\langle\operatorname{Ind}_{H}^{G} \eta_{j}, \chi\right\rangle_{G}=\left\langle\eta_{j},\left.\chi\right|_{H}\right\rangle_{H}$ and the previous table we verify that

$$
\begin{aligned}
& \text { Ind }_{H}^{G} \eta_{1}=\chi_{4} \oplus \chi_{5}, \\
& \text { Ind }_{H}^{G} \eta_{2}=\chi_{4} \oplus \chi_{6}, \\
& \text { Ind }_{H}^{G} \eta_{3}=\chi_{5} \oplus \chi_{6} .
\end{aligned}
$$

Let now $L$ be the cyclic group of order 4 generated by $b$ in $G$. Let $\nu$ be the irreducible character of $L$ defined by $\nu(b)=i$. The representation induced by $\nu$ in $G$ has degree $[G: L]=6$ and its decomposition in irreducible representations is

$$
\text { Ind }{ }_{L}^{G} \nu=\chi_{4} \oplus \chi_{5} \oplus \chi_{6} .
$$


Let $K$ be a number field with $\operatorname{Gal}(K / \mathbb{Q})=G$; such a field exists since $G$ is solvable (see Shafarevich [14], Th.7), and a possible realization of $K$ is as decomposition field of the polynomial $x^{8}+9 x^{6}+23 x^{4}+14 x^{2}+1$ (see the appendix in [8]). Let $K^{H}$ and $K^{L}$ be the subfields of $K$ fixed by $H$ and $L$, respectively. Hence, using well known properties of the Artin $L$-functions, by identities (2.8)-(2.11) we get that

$$
\begin{aligned}
& L\left(s, \nu \oplus \nu, K / K^{L}\right)=L\left(s, \nu, K / K^{L}\right)^{2} \\
& L\left(s, \nu \oplus \nu, K / K^{L}\right)=L\left(s, \eta_{1}, K / K^{H}\right) L\left(s, \eta_{2}, K / K^{H}\right) L\left(s, \eta_{3}, K / K^{H}\right)
\end{aligned}
$$

Note that $\nu$ and $\eta_{j}$ with $j=1,2,3$ are induced by 1 -dimensional characters, hence $L\left(s, \nu, K / K^{L}\right)$ and $L\left(s, \eta_{j}, K / K^{H}\right)$ are Hecke $L$-functions of finite order. Theorem 2 will therefore follow from (2.12) and (2.13) if we prove that $L\left(s, \nu, K / K^{L}\right)$ and $L\left(s, \eta_{j}, K / K^{H}\right)$ with $j=1,2,3$ are primitive in $\left\langle\mathbf{H}_{f}\right\rangle$.

Suppose now that $L\left(s, \nu, K / K^{L}\right)$ is not primitive in $\left\langle\mathbf{H}_{f}\right\rangle$. By the Artin reciprocity law we can identify each Hecke $L$-function of finite order with an Artin $L$-function associated with a 1 -dimensional character. Therefore there exist 1-dimensional characters $\tau_{i}$ of certain Galois extensions $K_{i} \hookrightarrow F_{i}, i=1, \ldots, r$, such that

$$
L\left(s, \nu, K / K^{L}\right)=L\left(s, \operatorname{Ind}_{L}^{G} \nu, K / \mathbb{Q}\right)=\prod_{i=1}^{r} L\left(s, \tau_{i}, F_{i} / K_{i}\right)
$$

There is no loss of generality if we assume that every $F_{i}$ is a Galois extension of $\mathbb{Q}$. Let $F$ be the compositum of all such fields and of $K$. Moreover, let $G_{i}=$ $\operatorname{Gal}\left(F_{i} / \mathbb{Q}\right), E_{i}=\operatorname{Gal}\left(F_{i} / K_{i}\right)$ and $E=\operatorname{Gal}(F / \mathbb{Q})$. By the properties of the Artin $L$-functions we can write (2.14) in the form

$$
\begin{aligned}
L\left(s, \operatorname{Lift}_{G}^{E} \operatorname{Ind}{ }_{L}^{G} \nu, F / \mathbb{Q}\right) & =L\left(s, \nu, K / K^{L}\right) \\
& =\prod_{i=1}^{r} L\left(s, \operatorname{Ind}_{E_{i}}^{G_{i}} \tau_{i}, F_{i} / \mathbb{Q}\right)=\prod_{i=1}^{r} L\left(s, \operatorname{Lift}_{G_{i}}^{E} \operatorname{Ind}_{E_{i}}^{G_{i}} \tau_{i}, F / \mathbb{Q}\right)
\end{aligned}
$$

and hence by (2.11) we obtain

$$
\operatorname{Lift}_{G}^{E} \chi_{4} \oplus \operatorname{Lift}_{G}^{E} \chi_{5} \oplus \operatorname{Lift}_{G}^{E} \chi_{6}=\operatorname{Lift}_{G}^{E} \operatorname{Ind}_{L}^{G} \nu=\bigoplus_{i=1}^{r} \operatorname{Lift}_{G_{i}}^{E} \operatorname{Ind}_{E_{i}}^{G_{i}} \tau_{i} .
$$

The $\chi_{j}, j=4,5,6$, are irreducible and non-monomial characters of $G$, hence each $\operatorname{Lift}_{G}^{E} \chi_{j}$ is irreducible and non-monomial as a character of $E$. Now we show that (2.15) is impossible if it is non-trivial, i.e. if $r>1$.

Suppose that $E_{i}=G_{i}$ for some $i$, for example for $i=1$. Then $\operatorname{Lift}_{G_{1}}^{E} \operatorname{Ind}_{E_{1}}^{G_{1}} \tau_{1}$ in (2.15) is 1-dimensional; but this is impossible, since the decomposition of Lift ${ }_{G}^{E}$ Ind ${ }_{L}^{G} \nu$ into irreducible characters is unique and the characters $\operatorname{Lift}_{G}^{E} \chi_{j}$ are 
irreducible and 2-dimensional; hence if (2.15) holds, then $\left[G_{i}: E_{i}\right] \geqslant 2$ for every $i$. Suppose now that $\left[G_{i}: E_{i}\right]=2$ for some $i$, for example for $i=1$. Then Lift $_{G_{1}}^{E}$ Ind ${ }_{E_{1}}^{G_{1}} \tau_{1}$ is 2-dimensional and irreducible (otherwise we fall into the previous

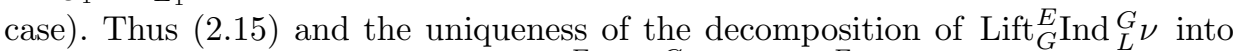
irreducible characters imply that $\operatorname{Lift}_{G_{1}}^{E}$ Ind $_{E_{1}}^{G_{1}} \tau_{1}=\operatorname{Lift}_{G}^{F} \chi_{j}$ for some $j \in\{4,5,6\}$. But this is impossible since every $\operatorname{Lift}_{G}^{E} \chi_{j}$ is non-monomial. Hence if (2.15) holds, then $\left[G_{i}: E_{i}\right] \geqslant 3$ for every $i$. Observing that the degree of $\operatorname{Lift}_{G}^{E} \operatorname{Ind}_{L}^{G} \nu$ is 6 we conclude that $n=2$ and $\left[G_{i}: B_{i}\right]=3$ for every $i$. If Lift ${ }_{G_{1}}^{E}$ Ind ${ }_{E_{1}}^{G_{1}} \tau_{1}$ is reducible then it contains a 1 -dimensional character (since $\operatorname{Lift}_{G_{1}}^{F}$ Ind $_{E_{1}}^{G_{1}} \tau$ is 3 -dimensional) which is impossible (since (2.15) shows that the irreducible components of $\operatorname{Lift}_{G}^{E}$ Ind ${ }_{L}^{G} \nu$ are 2-dimensional). As a consequence, Lift $G_{1}^{E}$ Ind ${ }_{E_{1}}^{G_{1}} \tau_{1}$ must be irreducible, but again this is impossible (the irreducible components of Lift $_{G}^{E}$ Ind ${ }_{L}^{G} \nu$ are 2-dimensional while $\operatorname{Lift}_{G_{1}}^{E}$ Ind $_{E_{1}}^{G_{1}} \tau_{1}$ is 3 -dimensional).

A similar argument can be used to prove that every $L\left(s, \eta_{j}, K / \mathbb{Q}\right)$ with $j=1,2,3$ is primitive in $\left\langle\mathbf{H}_{f}\right\rangle$, and Theorem 2 is proved.

It may be of interest to remark that the situation changes completely if we move from $\left\langle\mathbf{H}_{f}\right\rangle$ to $\mathcal{S}$, where the functions $L\left(s, \eta_{j}, K / K^{H}\right)$ and $L\left(s, \nu, K / K^{L}\right)$ are not any more primitive. In fact, identities (2.8)-(2.11) give the factorizations

$$
\begin{aligned}
L\left(s, \eta_{1}, K / K^{H}\right) & =L\left(s, \operatorname{Ind}_{H}^{G} \eta_{1}, K / \mathbb{Q}\right)=L\left(s, \chi_{4}, K / \mathbb{Q}\right) L\left(s, \chi_{5}, K / \mathbb{Q}\right) \\
L\left(s, \eta_{2}, K / K^{H}\right) & =L\left(s, \operatorname{Ind}_{H}^{G} \eta_{2}, K / \mathbb{Q}\right)=L\left(s, \chi_{4}, K / \mathbb{Q}\right) L\left(s, \chi_{6}, K / \mathbb{Q}\right) \\
L\left(s, \eta_{3}, K / K^{H}\right) & =L\left(s, \operatorname{Ind}_{H}^{G} \eta_{3}, K / \mathbb{Q}\right)=L\left(s, \chi_{5}, K / \mathbb{Q}\right) L\left(s, \chi_{6}, K / \mathbb{Q}\right) \\
L\left(s, \nu, K / K^{L}\right) & =L\left(s, \operatorname{Ind}_{L}^{G} \nu, K / \mathbb{Q}\right) \\
& =L\left(s, \chi_{4}, K / \mathbb{Q}\right) L\left(s, \chi_{5}, K / \mathbb{Q}\right) L\left(s, \chi_{6}, K / \mathbb{Q}\right)
\end{aligned}
$$

Such $L$-functions all belong to $\mathcal{S}$. In fact, it is known that the Hecke $L$-functions belong to $\mathcal{S}$; by the Artin reciprocity law the same holds true for every Artin $L$-function associated with a monomial representation, hence $L\left(s, \eta_{j}, K / K^{H}\right)$ with $j=1,2,3$ and $L\left(s, \nu, K / K^{L}\right)$ belong to the Selberg class. Moreover, the Langlands correspondence has been proved for irreducible 2-dimensional representations of solvable groups (the cyclic and dihedral cases by Artin himself via his reciprocity law, the tetrahedral case by Langlands [7] and the octahedral case by Tunnell [15]; see also Theorem 2.8 in [11]). Therefore, there exist cuspidal representations $\pi_{4}, \pi_{5}, \pi_{6}$ of $\mathrm{GL}_{2}\left(A_{\mathbb{Q}}\right)$ such that

$$
L\left(s, \chi_{j}, K / \mathbb{Q}\right)=L\left(s, \pi_{j}\right) \quad j=4,5,6 .
$$

This proves that also $L\left(s, \chi_{j}, K / \mathbb{Q}\right), j=4,5,6$, belong to $\mathcal{S}$ (being Artin $L$-functions, the Ramanujan bound holds true, while the analytic continuation comes from the known analytic properties of the $L$-functions associated with cuspidal representations). As a consequence, factorizations (2.12) and (2.13) are not longer distinct in $\mathcal{S}$. 
We finally remark that the primitivity in $\mathcal{S}$ of the functions $L\left(s, \chi_{j}, K / \mathbb{Q}\right)$ with $j=4,5,6$ can be proved unconditionally by the argument of Murty [9].

In fact, let $\sum_{n} a(n) n^{-s}$ be the Dirichlet series of $L\left(s, \chi_{4}, K / \mathbb{Q}\right)$. The Chebotarev density theorem implies that

$$
\sum_{p<x} \frac{|a(p)|^{2}}{p} \sim \log \log x
$$

(see [9]). By the known results on the structure of $\mathcal{S}$, if $L\left(s, \chi_{4}, K / \mathbb{Q}\right)=F_{1}(s) F_{2}(s)$ in $\mathcal{S}$ with $F_{1}(s), F_{2}(s) \neq 1$ identically, then $F_{1}(s), F_{2}(s)$ have degree 1 , hence $F_{j}(s)=L\left(s+i \theta_{j}, \zeta_{j}\right)$ for some Dirichlet characters $\zeta_{j}$ and real numbers $\theta_{j}$. The trivial zeros of $L\left(s, \chi_{4}, K / \mathbb{Q}\right)$ coming from the functional equations are real, so that $\theta_{1}=\theta_{2}=0$. Then

$$
\sum_{p<x} \frac{|a(p)|^{2}}{p}=\sum_{p<x} \frac{\left|\zeta_{1}(p)+\zeta_{2}(p)\right|^{2}}{p} \geqslant(2+o(1)) \log \log x,
$$

a contradiction.

\section{References}

[1] A.H. Clifford, Arithmetic and ideal theory of commutative semigroups, Ann. of Math. 39 (1938), 594-610.

[2] F. Halter-Koch, Halbgruppen mit Divisorentheorie, Exposition. Math. 8 (1990), 27-66.

[3] I.M. Isaacs, Character Theory of Finite Groups, Dover Publications 1994.

[4] H. Iwaniec, P. Sarnak, Perspectives on the analytic theory of L-functions, Visions in Mathematics Towards 2000, ed. by N. Alon et al., GAFA 2000 Special Volume, 705-741, Birkhäuser 2000.

[5] J. Kaczorowski, Axiomatic theory of L-functions: the Selberg class, In Analytic Number Theory, C.I.M.E. Summer School, Cetraro (Italy) 2002, ed. by A. Perelli and C. Viola, 133-209, Springer L.N. 1891, 2006.

[6] J. Kaczorowski, A. Perelli, The Selberg class: a survey, Number Theory in Progress, Proc. Conf. in Honor of A.Schinzel, ed. by K.Györy et al., 953-992, de Gruyter 1999.

[7] R.P. Langlands, Base Change for GL(2), Annals of Math. Studies 96, Princeton U. P. 1980.

[8] G. Malle, B.H. Matzat, Inverse Galois Theory, Springer Verlag 1999.

[9] M.R. Murty, Selberg's conjectures and Artin L-functions, Bull. A.M.S. 31 (1994), 1-14.

[10] M.R. Murty, V.K. Murty, Non-vanishing of L-Functions and Applications, Birkäuser Verlag 1997.

[11] M.R. Murty, An introduction to Artin L-functions, J. Ramanujan Math. Soc. 16 (2001), 261-307. 
[12] A. Perelli, A survey of the Selberg class of L-functions, part I, Milan J. Math. 73 (2005), 19-52.

[13] A. Perelli, A survey of the Selberg class of L-functions, part II, Riv. Mat. Univ. Parma (7) 3* (2004), 83-118.

[14] I.R. Shafarevich, Construction of fields of algebraic numbers with given solvable Galois group, Amer. Math. Soc. Transl. II Ser. 4 (1956), 185-237.

[15] J. Tunnell, Artin's conjecture for representations of octahedral type, Bull. Amer. Math. Soc. (N.S.) 5 (1981), 173-175.

Addresses: Jerzy Kaczorowski, Faculty of Mathematics and Computer Science, A.Mickiewicz University, 61-614 Poznań, Poland;

Giuseppe Molteni, Dipartimento di Matematica, Università di Milano, via Saldini 50, 20133 Milano, Italy;

Alberto Perelli, Dipartimento di Matematica, Università di Genova, via Dodecaneso 35, 16146 Genova, Italy

E-mail: kjerzy@amu.edu.pl; giuseppe.molteni@mat.unimi.it; perelli@dima.unige.it

Received: 10 October 2006 